\title{
Information Processing and Application System Development
}

\author{
Dr. Fan Yang, Prof. Zhenghong Dong, Mr. Jue Wang \\ Equipment Academic, Beijing, China
}

\begin{abstract}
This paper proposed a unified information processing and application system, which can automatically generate $\mathrm{C}++$ codes of math methods, and package the software and method into a standard component. Also, the system integrates different types of method in the unified information processing system with standard interface and format. Users can create new component and design test task with existing components. In the meantime, test results can be demonstrated in 2D or 3D format. The system is quite convenient for developers to develop new component, and easy for administrators to manage all existing component, and simple for users to design and operate test task, which saves them a lot of effort.
\end{abstract}

Keywords-software integration; automatic code generation; database management.

\section{INTRODUCTION}

The battle in the age of Information has very rapid tempo, high strength, and large scale. However, the military operation ability is limited to the ability of weapons and equipment. The types of existing weapons and equipment are various, software versions are complicate, and the data processing method database for supporting test task is very huge. Based on this kind of situation, the level of IT application of traditional test methods is far from enough, and the ability of dealing with complicate test tasks is weak. In order to improve the unified construction of test information process, it is necessary to integrate different kinds of test methods into a unified test platform, and to further optimize the organization of test information processing and application method. The idea of software integration [1, 2] reconstructs the existing software according to requirements, in order to implement the required technology with higher efficiency and lower costs. Users do not need to create the software again, and all software follows the same interface standard. In this way, software can be largely reused, and actually, software reuse technology is a very effective way to solve software crisis.

On the basis of unified test information processing system, according to the unified software development standard, standard method and software generation platform is constructed, which has the effect of leading, creation, and demonstration for the normalization, modularization, and standardization of test information processing and application. The unified test information processing system consists of 3 sub-platform, which are method and software generation platform, method and software management platform, and demonstration and verification platform. In general, this paper has made 3 major contributions,

(1) The proposed system integrates a wide range of test methods in different domains into a unified platform, so that users can directly use existing methods, rather than create them again.

(2) The method and software generation platform supports 3 different ways to generate program code. It can automatically generate $\mathrm{C}++$ code from Matlab code, or it can automatically generate $\mathrm{C}++$ code from math equation, or user can manually type the code. The way to generate code is flexible, and easy for users who do not have any programming skills.

The demonstration and verification platform applies various and rich visualization tools and environment to demonstrate the user interface as well as the data analysis results. It makes the UI friendly, and makes it is easy to understand the data analysis results.

\section{SYSTEM FUNCTION REQUIREMENT ANALYSIS}

From the perspective of system development, the system function can be concluded to the construction and development of 3 sub-platform, method and software generation platform, method and software management platform, and demonstration and verification platform. The system function framework is shown as figure 1 .

(1) Method and software generation platform.

This platform provides supporting tools, such as the development of method and software, simulation debug, and in-stockroom. This platform supports standard component generation which generates components with standard format and interface. It provides existing component database preview, new component/compound component project creation, new component/compound component analysis and preview, new component/compound component compiling and generation, new component/compound component test, new component/compound component in-stockroom and registration, and new component/compound component save. Actually, automatic code generation methods have been well developed. Example like reference [3], which gives a method to generate Matlab code from numerical algorithms. However, this platform supports 3 different ways to generate codes. It can automatically 


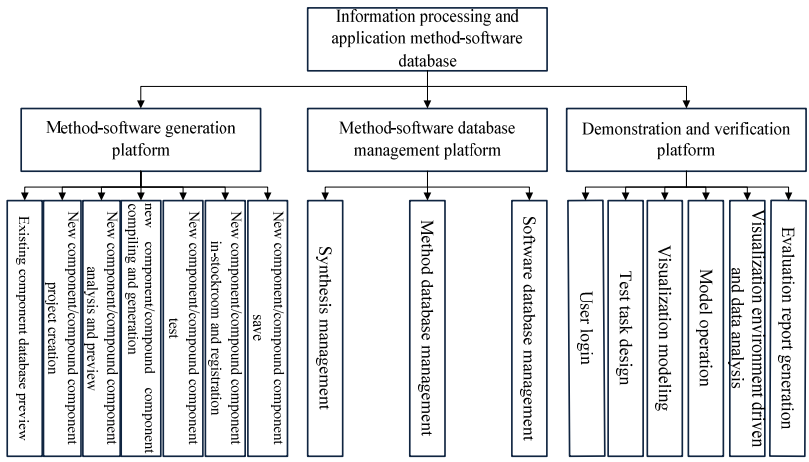

Figure 1. System function framework

generate $\mathrm{C}++$ code from Matlab code, or it can automatically generate $\mathrm{C}++$ code from math equation, or user can manually type the code.

(2) Method and software database management platform.

This platform provides application method, source code, store and management of component, which mainly includes the following 3 modules, synthesis management, method database management, and software database management. Synthesis management mainly provides task classification management, user authority management, component evaluation in-stockroom, component publish, online feedback and use-information statistic, information publish, and template management. Method and software management mainly provides the basic management of database (browse, search, edit, delete, export/import, and copy of database, maintenance and recovery), and version control.

(3) Demonstration and verification platform.

This platform mainly applies existing method database and software database to provide fast and typical case, visually construct and operate presentation environment. This platform includes the following 6 modules, user login, test task design, visualization modeling, model operation, visualization environment driven and data analysis, evaluation report generation. Test task design mainly provides test background, test control equipment and related index setting. Visualization modeling provides component visualization tree searching, visualize drag and drop and test modeling construction, the mapping between visualize graphics primitive and component dynamic depot, and component save. Model operation provides system assembling, operation dispatching, modeling debug, operational control, and data recording. Visualization environment driven and data analysis provides the visualization analysis of data, $2 \mathrm{D}$ or $3 \mathrm{D}$ test environment real-time display, and the evaluation report generation automatically generates different types of data, comparison results, and conclusion for demonstration and verification according to predefined template.

\section{SYSTEM DESIGN}

The system design includes the design for the 3 platforms as well, which are component generation, component management, and component application. The system information process framework is shown as figure 2.

The procedure of component generation has 3 steps.

(1) Component developers create new component or compound component, if it is a new component, then input the corresponding equation or program codes. If it is a compound component, then drag an existing component from the component tree, and add a self-defined visualized module. At this point, it is OK to input the corresponding equation or program codes as well. After the component is created, by the code analysis, document and code preview is generated. After compelling, the correspondent algorithm document and excusable software component (*.dll/*.so) are generated. The document consists of component description, equation, and codes.

(2) Testers use document checking tools to check the content and format of documents, use test tools and test examples to carry on function test and performance test. Performance test checks the semantic and grammar of component, and function test assesses if the function meet up with the requirement of design, and return the test results to component developer for revising.

(3) If test is passed, developers submit component instockroom registration, and submit all document, code and executable software.

The procedure of component management has the following 2 steps,

(1) Managers verify the in-stockroom application, and publish information if there is no error. Managers can select the published target.

(2) Manager can carry on synthesis management, method database management, and software database management. Synthesis management includes task classification management, user authority management, component verification in-stockroom, user assessment and use-information statistic, information publish and external visiting management, and template management. Method and software based save and management provides the basic management of database, and version control.

The procedure of component application has the following 3 steps.

(1) Create test task design, which mainly provides test background, test and control equipment, and related test index setting.

(2) Carry on visualization modeling, based on the visualized tree structure to carry out component search, drag and drop and test model construction, the mapping between visualized graphic primitive and executable component dynamic base, and component save.

(3) Execute model dispatch, carry out model debug, and operational control, record operation data, drive visualization environment, analyze operational data, and generate evaluation report. 




Figure 2. System information processing framework

\section{PRototype Design}

According to the system framework built above, this paper has provided the system prototype design in figure 4-(1-6). Figure 4-(1-2) show the method and software generation platform. Figure 4-1 shows the math equation typing template, where user can type any complicate math equations using the equation editor. Figure 4-2 shows the compound component editor, where compound component can be created based on existing component and self-defined component. Figure 4-(3-4) show the method and software database management platform. Figure 4-3 shows the synthesis management, which supports task classification management, user authority management, component evaluation in-stockroom, component publish, online feedback and use-information statistic, information publish, and template management. Figure 4-4 shows the method database management, which supports the basic database management function, such as browse, search, edit, delete, export/import, and copy of database, maintenance and recovery. Figure 4-(56 ) show the demonstration and verification platform. Figure 4-5 shows the task design interface, where user can directly drag a component from the component tree on the left side of the page, and drop it in the design window, as well as connecting related component. Figure 4-6 shows the analysis results in $2 \mathrm{D}$ and $3 \mathrm{D}$. The $2 \mathrm{D}$ results show information like trajectory comparison, error analysis, etc. and the 3D shows the object's trajectory.

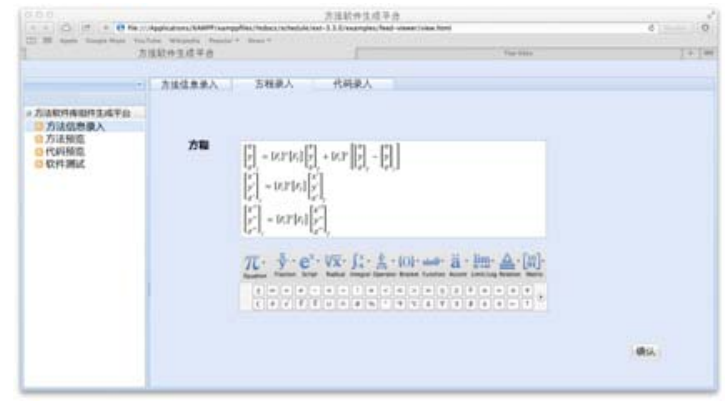

Figure 4-1. Equation information input interface

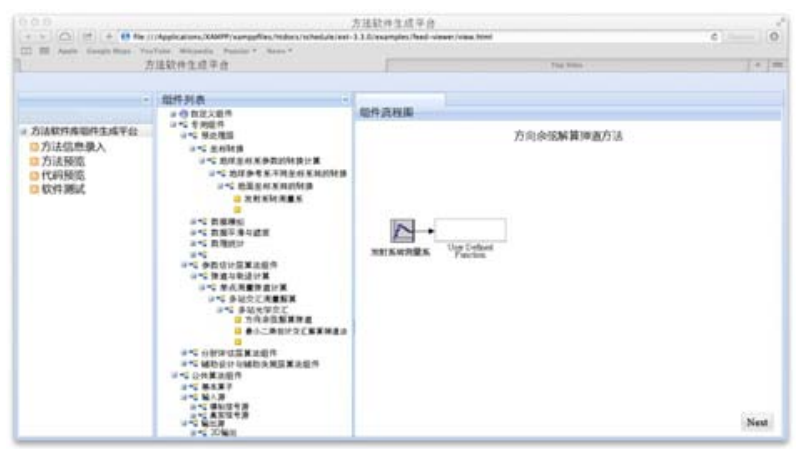

Figure 4-2. Compound component edit interface

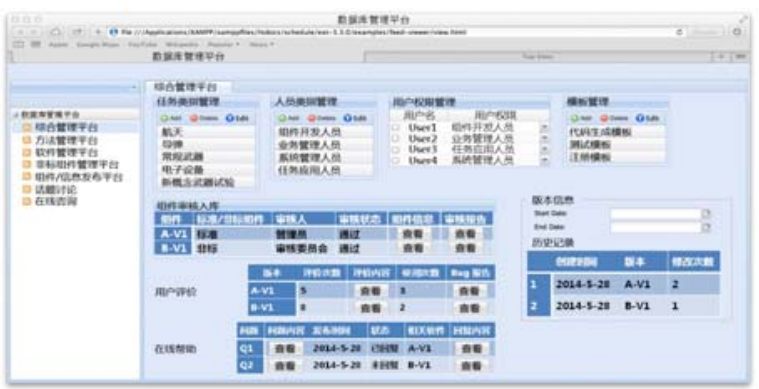

Figure 4-3. Method and software management platform (Synthesis management)

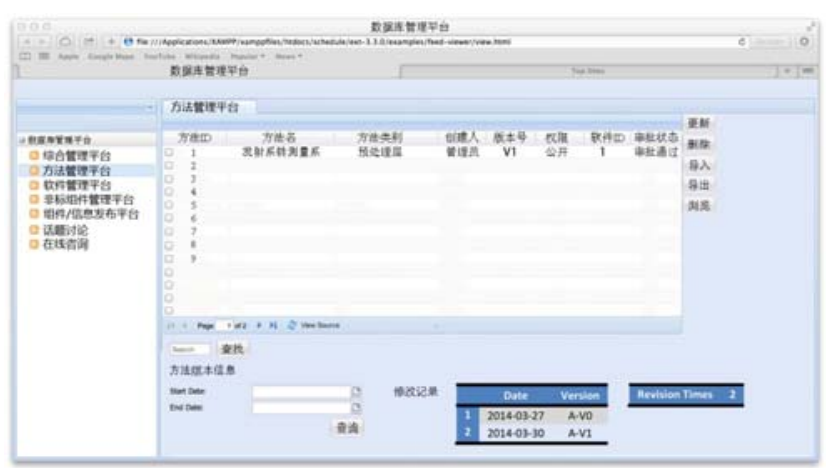

Figure 4-4. Method and software management platform (Method database management) 


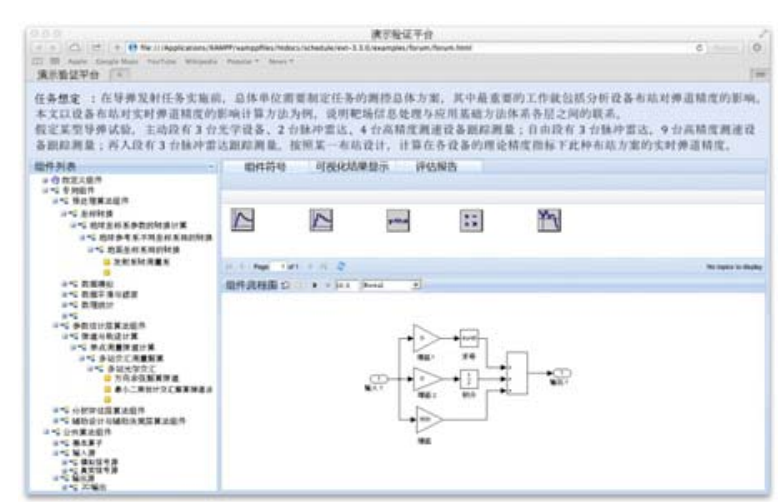

Figure 4-5. Visualization modeling and simulation operation

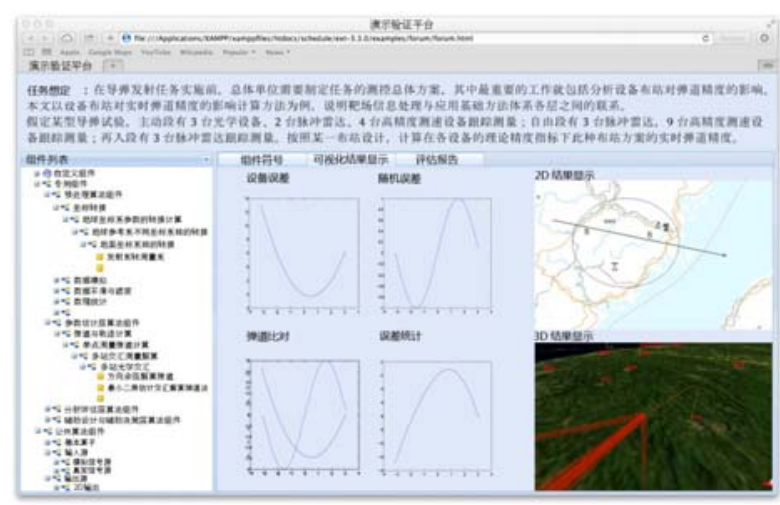

Figure 4-6. Visualization results analysis and 3D environment driven

\section{CONCLUSION}

This paper has proposed the system development of information processing and application, which includes the development of three sub-platforms, method and software generation platform, method and software database management platform, and demonstration and verification platform. This paper also provides the system design of the framework, and gives the prototype design of the whole system. This system integrates all kinds of methods related to the test task, where user can develop new method in the platform, or directly apply the existing methods to design test task. Especially, user can just simply type the math equation, and the codes can be automatically generated. Also, the database management platform manages the standard and non-standard methods and software. The demonstration and verification platform supports the visualized design of test task, and data processing results in both $2 \mathrm{D}$ and $3 \mathrm{D}$, which improves the operability of test design, and also improves the understandability of data processing results.

\section{REFERENCES}

[1] T. Suzudo, K. Nabeshima, and H. Takizawa, "Software Integration for Monitoring Systems With High Flexibility", Progress in Nuclear Energy, 43(1-4): 405-411, 2003.

[2] P. Masiakowski, and S. Wang, "Integration of software tools in patent analysis”, World Patent Information, 35: 97-104, 2013.

[3] B. A. Marsofl, K. A. Gallivan, and H. A. G. Wijshoff, "The Utilization of Matrix Structure to generate Optimized Code from MATLAB Programs”, International Journal of Parallel Programming , 27(2): 73-96, 1999. 